\title{
THE LOCATIONS OF THE CORE AND CONAL EMISSION REGIONS IN AN INVERSE-COMPTON SCATTERING MODEL
}

\author{
G. J. QIAO \\ Center of Astronomy and Astrophysics, CCTS (World Laboratory) and Department of Geophysics, Peking University \\ C. G. Li AND M. Li \\ Department of Geophysics, Peking University
}

\begin{abstract}
The physical conditions and locations of the emission regions for core and hollow cone emissions are very important in understanding the mechanism of radio pulsars. We present two related methods in an Inverse-Compton Scattering (ICS) model in this paper, which give a clear scenario for determining the location of the emission regions and are consistent with the results given by Cordes et al. (1984) and Rankin (1990).
\end{abstract}

\section{Introduction}

A successful magnetospheric theory would specify the physical conditions within the region of flow and would lead to well constrained predictions concerning the radio emission. But, lacking such a theory, efforts have been made to identify the emission mechanism with the hope of working backwards toward an understanding of the physical conditions in the magnetosphere (Taylor and Stinebring 1986).

Some authors have attempted to place observational constraints on the altitude from which the radio emission arises (Cordes 1978; Cordes and Stinebring 1984; Kardashev et al. 1982; Matese and Whitmire 1980). Cordes and his co-workers have placed limits on the emission radius $\rho<0.01 R_{\mathrm{LC}}$, where $R_{\mathrm{LC}}=c P / 2 \pi$ is the light-cylinder radius. Rankin (1990) describes an effort to define the geometric properties of the core emission region and concludes that the core emission appears to come essentially from the stellar surface, filling the entire polar-cap "gap" region.

Here, we give a calculation within the framework of an inverse-Compton scattering (ICS) model, which is intended both to determine the locations of the core and conal emission regions and to define some other physical properties of the emission region.

\section{The ICS model}

An ICS model has been proposed by Qiao (1988a,b, 1992). The idea is that when the discharge in the inner gap takes place-which would be associated with a low frequency wave - the photons of this lowfrequency wave will acquire energy from the relativistic particles via the ICS process. The outgoing photons are the ones that we observe. In this situation, the location of the emission region can be calculated from physical and geometrical considerations. Here, we use two methods, one which uses the "absorption" phenomenon described by Rankin (1983b), and another which is a purely theoretical calculation.

\section{The "absorption" method of locating the emission regions}

It is assumed that the magnetic field is purely dipolar, which can be described as

$$
\rho=r_{\mathrm{e}} \cos ^{2} \varphi,
$$

where $(\rho, \varphi)$ is a point on the magnetic field. $r_{\mathrm{e}}$ is a parameter which is different for different field lines. At any point $(\rho, \varphi)$, the tangent line is

$$
\tan \varphi=\frac{1}{4}\left(3 \cot \theta \pm \sqrt{9 \cot ^{2} \theta+8}\right),
$$

$\theta$ is the angle between the tangent line and the magnetic axis. For the distance $R^{\prime}$ (figure 1 ), we have

$$
R^{\prime}=r_{\mathrm{e}} \frac{\cos ^{2} \varphi}{\sin \varphi}
$$

When $\theta_{\mathrm{c}}=(R \Omega / c)^{\frac{1}{2}} \ll 1$ we have

$$
R^{\prime} \cong R\left(1+\theta_{\mathrm{c}} \cot \theta\right) \text {. }
$$

We assume

$$
\theta=\frac{1}{2}\left(W_{1}+W_{2}\right)
$$

where $W_{1}\left(W_{2}\right)$ is the pulse width corresponding to the upper (lower) part of the absorption region (Rankin 1983). 


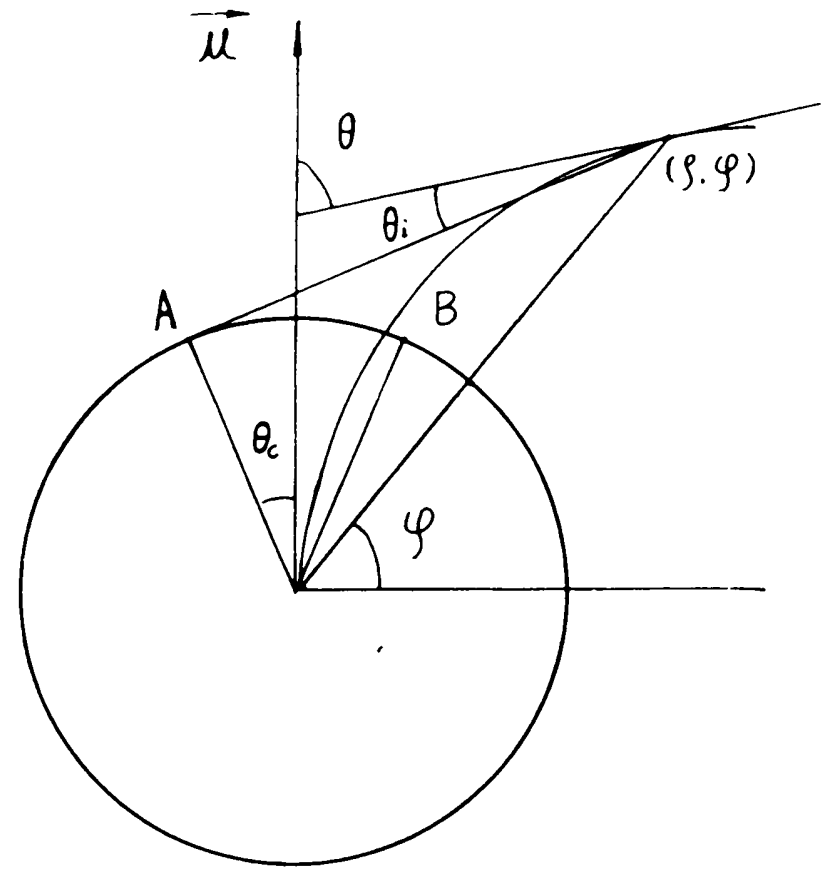

Figure 1 The ICS geometry. As the sparking takes place around the cap, the low frequency wave is emitted from point $A$ to point $B$ equivalently. The low frequency wave is scattered by the pairs moving along the magnetic field lines. Line MN is the tangent to the magnetic field line at point $\mathrm{N}(\rho, \varphi)$. MN is the direction of the observed emission.

From eq.(5) and the observations (Rankin 1983), we calculate $\theta$ which we substitute $\theta$ into eq.(2) to find $\varphi$, then we obtain $\rho$ from eq.(1). For conal single profiles we take $r_{\mathrm{e}}=R_{\mathrm{LC}}$, where $R_{\mathrm{LC}}=c P / 2 \pi$ is the radius of the light cylinder. The results for conal single pulsars are shown in table 1.

Table 1 The emission region of pulsars with conal single profiles

\begin{tabular}{|cccccc|}
\hline PSR & $\begin{array}{c}\theta \\
\left({ }^{\circ}\right)\end{array}$ & $\begin{array}{c}P \\
(\mathrm{~s})\end{array}$ & $\begin{array}{c}R_{\mathrm{LC}} \\
\left(10^{10}\right.\end{array}$ & $\begin{array}{c}\rho \\
\left(10^{8}\right.\end{array}$ & $\rho / R_{\mathrm{LC}}$ \\
$\mathrm{cm})$ & $\mathrm{cm})$ & \\
\hline $0031-07$ & 14.0 & 0.943 & 0.45 & 1.2 & 0.027 \\
$0126+28$ & 5.5 & 0.558 & 0.27 & 0.11 & 0.037 \\
$0628-28$ & 13.0 & 1.244 & 0.59 & 1.3 & 0.022 \\
$0809+74$ & 16.5 & 1.292 & 0.62 & 2.2 & 0.035 \\
\hline
\end{tabular}

For core-single and/or triple profile pulsars, eq.(5) is used to calculate $\theta$; then from eq.(4) and eq.(2) it is possible to find $R^{\prime}$ and $\varphi$. Eq.(3) is then used to get $r_{\mathrm{e}}$, and finally we find $\rho$ from eq.(1). Results for several pulsars are shown in table 2.

The calculations above show that the core emission region is close to the surface of the neutron star. The conal emission region is far from the neutron star. The values compare well with the limit determined for PSR $0525+21$ by Cordes et al. (1983), $r<0.06 R_{\mathrm{LC}}$. This limit is also in agreement with that given by Qiao (1992).
Table 2 The emission region of pulsars with core-single or triple profiles.

\begin{tabular}{|ccccccc|}
\hline PSR & $\begin{array}{c}\theta \\
\left(^{\circ}\right)\end{array}$ & $\begin{array}{c}P \\
(\mathrm{~s})\end{array}$ & $\begin{array}{c}R_{\mathrm{LC}} \\
\left(10^{10}\right. \\
\mathrm{cm})\end{array}$ & $\begin{array}{c}r_{\mathrm{e}} \\
\left(10^{10}\right. \\
\mathrm{cm})\end{array}$ & $\begin{array}{c}\rho \\
\left(10^{6}\right.\end{array}$ & $\rho / R_{\mathrm{LC}}$ \\
$\mathrm{cm})$ & \\
\hline $0329+54$ & 2.33 & 0.725 & 0.34 & 0.58 & 4.2 & 0.0007 \\
$1449-64$ & 4.38 & 0.179 & 0.085 & 0.17 & 4.4 & 0.0052 \\
$1642-03$ & 2.45 & 0.388 & 0.19 & 0.57 & 4.6 & 0.0024 \\
$1749-28$ & 3.30 & 0.563 & 0.27 & 0.29 & 4.3 & 0.0016 \\
\hline
\end{tabular}

\section{The location of the emission re- gions in the ICS model.}

In the calculations pertaining to the ICS model (Qiao 1992), the central or core-emission beam and hollow cone emission beam can be modeled at different frequencies. Consequently, the location of the emission region can be determined. In different regions, the ICS cross section is different.

$$
\sigma(1)=\frac{\left(1-\beta \cos \theta_{i}\right) \sin ^{2} \theta_{i}}{\gamma^{2}\left(1+\beta \cos \theta_{i}\right)^{2}}
$$

where $\theta_{\mathrm{i}}$ is the angle between the directions of the incoming photons and the magnetic field and $\gamma$ is the Lorentz factor. In our calculation, regions are ignored wherein the cross section $\sigma(1)<\frac{1}{2} \sigma_{\max }$. The results are shown in figure 2 and table 3 .

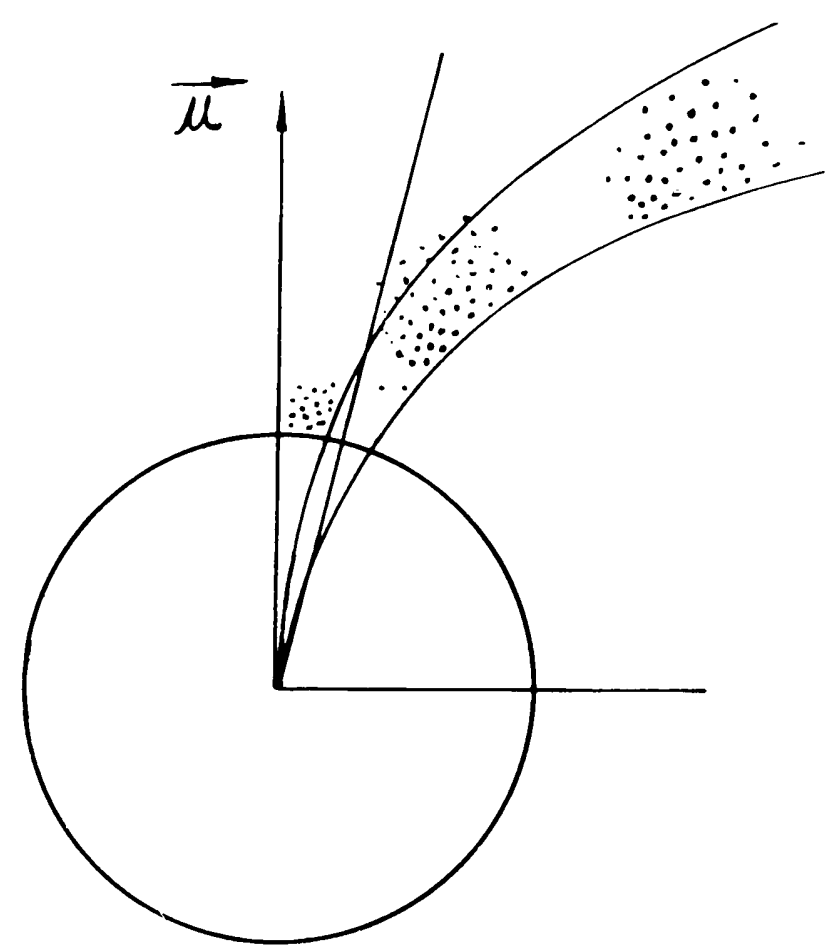

Figure 2 The location of the observed emission regions for a given frequency. 
Table 3 The location of the emission region in the ICS Model (for all frequencies)

\begin{tabular}{|ccccc|}
\hline PSR & $\begin{array}{c}\rho \\
\left({ }^{\circ}\right)\end{array}$ & $\begin{array}{c}\rho \\
\left(10^{\circ} \mathrm{cm}\right)\end{array}$ & core & cone \\
\hline $1642-03$ & $0.5-1.8$ & $1.2-7.5$ & $* * *$ & \\
$1933+16$ & $0.5-2.3$ & $1.2-18.3$ & $* * *$ & \\
$0809+74$ & $2-40$ & $7.5-1700$ & & $* * *$ \\
$2026+28$ & $3-40$ & $3.2-330$ & & $* * *$ \\
\hline
\end{tabular}

\section{Discussion}

1. The location of the core emission region is very close to the surface of the neutron star as suggested by Rankin (1990). In our calculations, the core emission is emitted from a region near the center of the polar-cap gap region.
2. The location of the hollow cone emission is divided into two parts. The inner hollow cone is closer to the surface of the neutron star, but farther than the region where the core emission is emitted for a given pulsar. The outer hollow cone is furthest from the stellar surface.

3. The two methods discussed here are consistent. The first one (using the absorption data) gives a location which is in or near the center of the total emission region. The second method gives the total emission region; in fact, in this region, the intensity of the emission is not the same. For example, the core emission is more efficient at a place close to the surface of the neutron star.

Acknowledgments: The authors would like to thank Prof. J. M. Rankin and Mr. Y. D. Zhu for helpful discussions. They would also like to thank Mr. G. Q. Li for his help in preparing this work. This project was supported by the Natural Science Foundation of China. 